3-2005

\title{
A Wavelet-Based Multiresolution PID Controller
}

\author{
Shahid Parvez \\ ControlSoft Inc., Willoughby Hills, sparvez@controsoftinc.com \\ Zhiqiang Gao \\ Cleveland State University, Z.GAO@csuohio.edu
}

Follow this and additional works at: https://engagedscholarship.csuohio.edu/enece_facpub

Part of the Controls and Control Theory Commons

How does access to this work benefit you? Let us know!

\section{Publisher's Statement}

(C) 2005 IEEE. Personal use of this material is permitted. Permission from IEEE must be obtained for all other uses, in any current or future media, including reprinting/republishing this material for advertising or promotional purposes, creating new collective works, for resale or redistribution to servers or lists, or reuse of any copyrighted component of this work in other works.

\section{Original Citation}

Parvez, S., \& Zhiqiang, G. (2005). A wavelet-based multiresolution PID controller. IEEE Transactions on Industry Applications, 41, 2, 537-543.

\section{Repository Citation}

Parvez, Shahid and Gao, Zhiqiang, "A Wavelet-Based Multiresolution PID Controller" (2005). Electrical Engineering \& Computer Science Faculty Publications. 104.

https://engagedscholarship.csuohio.edu/enece_facpub/104

This Article is brought to you for free and open access by the Electrical Engineering \& Computer Science Department at EngagedScholarship@CSU. It has been accepted for inclusion in Electrical Engineering \& Computer Science Faculty Publications by an authorized administrator of EngagedScholarship@CSU. For more information, please contact library.es@csuohio.edu. 


\title{
A Wavelet-Based Multiresolution PID Controller
}

\author{
Shahid Parvez and Zhiqiang Gao, Member, IEEE
}

\begin{abstract}
A novel controller based on multiresolution decomposition using wavelets is presented. The controller is similar to a proportional-integral-derivative (PID) controller in principle and application. The output from a motion control system represents the cumulative effect of uncertainties such as measurement noise, frictional variation, and external torque disturbances, which manifest at different scales. The wavelet is used to decompose the error signal into signals at different scales. These signals are then used to compensate for the uncertainties in the plant. This approach provides greater expanse over the degree of control applied to the system. Through hardware and simulation results on motion control systems, this controller is shown to perform better than a PID in terms of its ability to provide smooth control signal, better disturbance, and noise rejection.
\end{abstract}

Index Terms-Motion control, multiresolution, wavelet controller, wavelets.

\section{INTRODUCTION}

$\mathbf{T}$ HE wavelet transform, developed earlier as a mathematical tool [2], [6], [7], has in recent years been used in various industrial applications. Wavelets possess two features that make them especially valuable for data analysis: they reveal local properties of the data and they allow multiscale analysis. Their locality is useful for applications that require online response to changes, such as controlling a process. Recently, some work has been reported on use of time-frequency localization of wavelet transforms in process control industry [1], [3], [4]. However, these applications have been largely limited to signal processing that indirectly help to improve the performance of the control systems. In this paper, a direct application of wavelet transform to controller design is proposed. In particular, based on the multiresolution decomposition property of wavelet, a wavelet controller analogous to the well-known proportional-integral-derivative (PID) controller is presented.

The PID controller has been around for more than 80 years [8] and is still widely used across the industry. In addition to its effectiveness as a control means, the reasons for its longevity can be traced to the fact that it is easy to implement in analog circuits and is intuitive, which makes it easy to tune. On the other hand, this very simplicity limits the performance of PID controllers. Various attempts have been made to enhance the

S. Parvez is with ControlSoft Inc., Willoughby Hills, OH 44092 USA (e-mail: shahid402@yahoo.com; sparvez@controsoftinc.com).

Z. Gao is with the Center for Advanced Control Technologies, Fenn College of Engineering, Cleveland State University, Cleveland, OH 44115 USA (e-mail: z.gao@csuohio.edu).
PID controller, such as the nonlinear PID, or PID with nonlinear gains. See, for example, nonlinear PID (NPID) controller in [9]. The wavelet transform provides another vehicle in developing high performance controllers based on the PID principles.

In general, a PID controller takes as its input the error $(e)$ and acts on the error to generate a control output $(u)$, as shown in the following equation:

$$
u=k_{p} e+k_{I} \int e d t+k_{D} \frac{d e}{d t}
$$

where $k_{p}, k_{I}$, and $k_{D}$ are the PID gains to be selected. The three terms in this controller intuitively represent the current, the past, and the trend of the error. Arguably, it is this error-based design philosophy that has made the PID a successful controller in industrial settings, where the plant is often highly nonlinear, uncertain, and time-varying.

The controller in (1) can be described as linear PID since $u$ is a weighted sum of the error, the integration of the error, and the derivative of the error. One type of proposed improvements is to use nonlinear gains in PID, which make it more powerful and flexible [9]. In this case, the control law is described as

$$
u=k_{p} g_{p}(e)+k_{I} \int g_{I}(e) d t+k_{D} \frac{d}{d t} g_{D}(e)
$$

where $g_{p}(),. g_{I}($.$) , and g_{D}($.$) are certain nonlinear functions.$

In this paper, a generalization of the PID controller, based on multiresolution decomposition of the error by using small waves popularly known as wavelets, is presented. The basic idea is that, if $e$ is decomposed as

$$
e=\sum_{i} e_{i}
$$

where $e_{i}$ is the error signal at the $i$ th scale, then a generalized PID controller can be formulated as

$$
u=\sum_{i} k_{i} e_{i}
$$

where $k_{i}$ are controller parameters to be determined.

Applying wavelet decomposition to error signal $e$ extracts the average energy of the error signal, as well as the cumulative effect of many underlying phenomena such as process dynamics, measurement noise, and effects of external disturbances, which manifest on different scales (3). Then, each one of these components is scaled by its respective gain and then added together to generate the control signal $(u)(4)$. Note that the wavelet decomposition provides much higher resolution than a traditional PID in describing the history and predicting the immediate future of the error. Therefore, this controller is named a multiresolution proportional-integral-derivative (MRPID) controller. 
In Section II, a brief description of wavelet transforms and multiresolution decomposition is given. The framework of an MRPID is discussed in Section III. Implementation issues are discussed in Section IV. Implementation of the MRPID on motion control systems and experimental results are given in Section V and VI. Finally, some concluding remarks are included in Section VII.

\section{WAVELETS AND MULTIRESOLUTION DECOMPOSITION}

Multiresolution analysis is a convenient framework for hierarchical representation of functions or signals on different scales. The basic idea of multiresolution analysis is to represent a function as a limit of successive approximations. Each of these successive approximations is a smoother version of the original function with more and more of the finer "details" added. Wavelets are terminating basis vectors which are used to represent a signal using a set of coefficients. Consider a sampled signal $f(x)$ and generate the following sequence of approximations [2]:

$$
\begin{aligned}
f^{m}(x) & =\sum_{n=-\infty}^{\infty} f_{m, n} \phi\left(2^{m} x-n\right)_{m=0,1,2, \ldots} \\
f(x) & =\sum_{m} f^{m}(x) .
\end{aligned}
$$

Each approximation is expressed as the weighted sum of the shifted versions of the same function $\phi(x)$, which is called the scaling function. If the $(m+1)$ th approximation is required to be a refinement of the $m$ th approximation, then the function $\phi\left(2^{m} x\right)$ should be a linear combination of the basis functions spanning the space of the $(m+1)$ th approximation, i.e.,

$$
\phi\left(2^{m} x\right)=\sum_{k} h(k) \phi\left(2^{m+1} x-k\right) .
$$

If $V^{m+1}$ represents the space of all functions spanned by the orthogonal set $\left\{\phi\left(2^{m+1} x-k\right) ; k \in Z\right.$, the set of integers $\}$ and $V^{m}$ the space of the coarser functions spanned by the orthogonal set $\left\{\phi\left(2^{m} t-p\right) ; p \in Z\right\}$, then $V^{m} \subset V^{m+1}$. Let

$$
V^{m+1}=V^{m} \oplus W^{m} .
$$

Then $W^{m}$ is the space that contains the information added upon moving from the coarser $f^{m}(x)$ to the finer $f^{m+1}(x)$ representation of the original function $f(t)$. Mallat [2] shows that $W^{m}$ 's are spaces that are spanned by the orthogonal translates of a single function $\psi\left(2^{m} x\right)$, thus leading to the following equation:

$$
f^{(m+1)}(x)=f^{m}(x)+\sum_{n=-\infty}^{\infty} f_{m, n} \psi\left(2^{m} x-n\right) .
$$

The function $\psi(x)$ is called a wavelet and is related to the scaling function $\phi(x)$, through the following relationship:

$$
\psi\left(2^{m} x\right)=\sum_{k} g(k) \phi\left(2^{m+1} x-k\right) .
$$

TABLE I

Coefficients of DAubechies (4) FiLters

\begin{tabular}{lllll}
\hline $\mathrm{h}$ & 0.4830 & 0.8365 & 0.2241 & -0.1294 \\
\hline $\mathrm{g}$ & -0.1294 & -0.2241 & 0.8365 & -0.4830 \\
\hline \hline
\end{tabular}

$h(k)$ and $g(k)$ form a conjugate mirror filter pair. Concluding the discussion, a mixed-form $N$-level discrete wavelet series representation of the signal $f(x)$ is given by

$$
\begin{aligned}
f(x) & =\sum_{k} c_{N, k} \phi_{N, k}(x)+\sum_{m=1}^{N} \sum_{k} d_{m, k} \psi_{m, k}(x) \\
c_{m, k} & =\sum_{k} f(x) \overline{\phi_{m, k}(x)} \\
d_{m, k} & =\sum_{k} f(x) \overline{\psi_{m, k}(x)}
\end{aligned}
$$

where $\bar{\phi}(x)$ and $\bar{\psi}(x)$ are the conjugate functions corresponding to $\phi(x)$ and $\psi(x)$, respectively. Interestingly, the multiresolution concept, besides being intuitive and useful in practice, forms the basis of a mathematical framework for wavelets. One can decompose a function into a coarse version plus a residual and then iterate this to infinity. If properly done, this can be used to analyze wavelet schemes and derive the wavelet basis. It can be seen from (10) that a wavelet transform decomposes a signal $f(x)$ into trend $(c)$ and detail coefficients $(d)$. An efficient approach in computing the discrete wavelet transform (DWT) is to use the subband coding scheme [10] which uses only the filters $h(k)$ and $g(k)$, which are found to be

$$
\begin{aligned}
& h(k)=\sqrt{2} \sum_{x} \phi(x) \overline{\phi(2 x-k)} \\
& g(k)=\sqrt{2} \sum_{x} \psi(x) \overline{\psi(2 x-k)} \\
& g(k)=(-1)^{k} \overline{h(-k+1) .}
\end{aligned}
$$

Equations (10) and (11) provide a hierarchical and fast scheme for the computation of the wavelet coefficients of a given function. A pair of the "Daubechies" (4) filters used in experiments is shown in Table I.

\section{A. Decomposition Process}

The DWT of a signal $f(x)$ results in trend $(c)$ and detail coefficients $(d)$ as given by (10). The first step in signal decomposition consists of computing these trend and detail coefficients. Thereafter, the trend coefficients combined with the scaling function as a basis is used to generate the trend signal [the left-hand side of the summation in (10)] and the detail coefficients using the wavelets as a basis are used to generate the detail signals [the right-hand side of the summation in (10)]. The trend signal captures the high-scale (low-frequency) information and the detail signal captures the low-scale (high-frequency) information contained in the signal $f(x)$. Depending upon the number of decomposition levels, the end product of a multiresolution decomposition is a set of these signals at different scales (frequencies), as shown in (12), where $f_{H}$ is the high-scale signal, $f_{L}$ is the low-scale signal, 


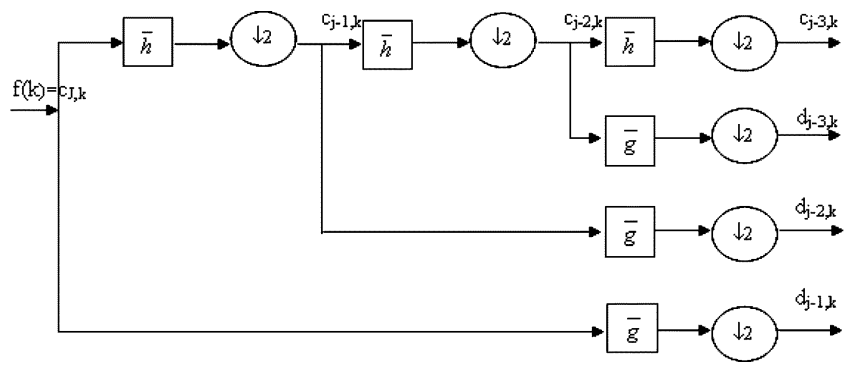

Fig. 1. Decomposition analysis.

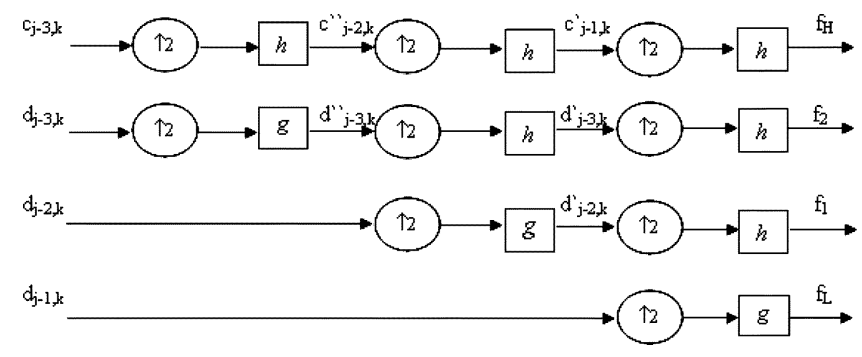

Fig. 2. Decomposition synthesis.

and $f_{M i}, i=1, \ldots N-1$, are the medium-scale signals where $N$ is the number of decomposition levels. For example, if a three-level decomposition of error signal is done, it results in one trend signal (low frequency) and three detail signals (high and intermediate frequency). There is redundancy in the trend signal; hence, only one obtained at the last level is chosen. The frequency information of these decomposed signals is approximate since the decomposition process does not use a precise frequency-characterized basis vector such as sines and cosines which are used in Fourier analysis

$$
f(x)=f_{H}(x)+f_{M_{1}}(x)+\ldots+f_{M_{N-1}}(x)+f_{L}(x) .
$$

The process of decomposition uses a subband coding scheme that is illustrated in Figs. 1 and 2. The DWT can be computed using the filters $\bar{h}(k)$ and $\bar{g}(k)$ which form a quadrature conjugate mirror filter pair with $h(k)$ and $g(k)$, which are given by (11). Fig. 1 illustrates the analysis part of a three-level decomposition scheme using subband coding. The result of the analysis step is a set of intermediate coefficients, which represent the weights of the original signal in terms of the basis functions used, namely the scaling function and the wavelet function. The original sampled signal is filtered with the scaling function and the wavelet function and downsampled by two, resulting in the trend and detail coefficients at level one. The trend coefficients thus obtained are then used as the original signal and filtered with the scaling function and the wavelet to yield the coefficients at level two. This process is repeated depending upon the number of decomposition levels desired.

The synthesis process involves upsampling the coefficients obtained during the analysis step by a factor of two and filtering them with the corresponding reconstruction filters. The synthesis process for a three-level decomposition is shown in Fig. 2. The resultant signals are the trend signal $f_{H}$, the detail signal $f_{L}$, and the intermediate resolution signals $f_{1}$ and $f_{2}$.

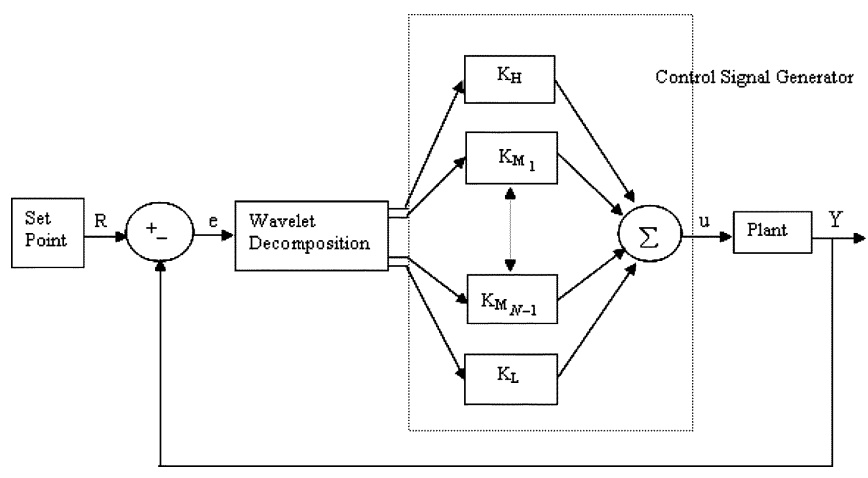

Fig. 3. Block diagram of an MRPID.

\section{MRPID CONTROLLER}

In general, a PID controller takes as its input the error $e$ and then acts on the error to generate a control output $u$, as shown in (1). Gains $K_{P}, K_{I}$, and $K_{D}$ are the proportional, integral, and derivative gains used by the system to act on the error, the integral of the error, and the derivative of the error, respectively. In terms of frequency information, the proportional and integral terms tend to capture the low-frequency information of the error signal and derivative captures the high-frequency information of the signal. In a similar manner, an MRPID decomposes the error signal into its high-, low-, and intermediate-scale components, as shown in (12). Each of these components are scaled by their respective gains and then added together to generate the control signal $u$ as

$$
u=K_{H} e_{H}+K_{M_{1}} e_{M_{1}}+\ldots+K_{M_{N-1}} e_{M_{N-1}}+K_{L} e_{L} .
$$

Unlike a PID controller, which has three tuning parameters ( $K_{P}, K_{I}$, and $K_{D}$ ), an MRPID can have two or more parameters based on the number of decomposition levels performed on the error signal. For example, a one-level decomposition yields a low- and a high-frequency component. So, a controller with one-level decomposition has two gains. In order to achieve higher resolution if a two-level decomposition is done on the error signal, three signal components of $e$ are generated. Each one of these components can be scaled by a gain and added to generate the control signal. A schematic diagram of a plant using an MRPID is shown in Fig. 3. Since there are a number of different wavelets, the choice of a wavelet affects the performance of the controller. In general, there are two kinds of choices to make: the system of representation (continuous or discrete) and the properties of the wavelets themselves: for example, the number of degree of regularity. A common theme in choice is tradeoff. If more resolution in frequency is desired, less resolution in time is achieved; if more vanishing moments are required, the size of the wavelet has to be increased. In the examples that are discussed in this paper, "Daubechies" of order 4 were found to be suitable for controls. For more details on the selection of wavelets, see [10].

All physical systems are subjected to some type of extraneous signals or noise during operation. Therefore, in the design of a control system, consideration has to be made that the system provides greater insensitivity to noise and disturbance. The effect of feedback on noise and disturbance greatly depends on where 

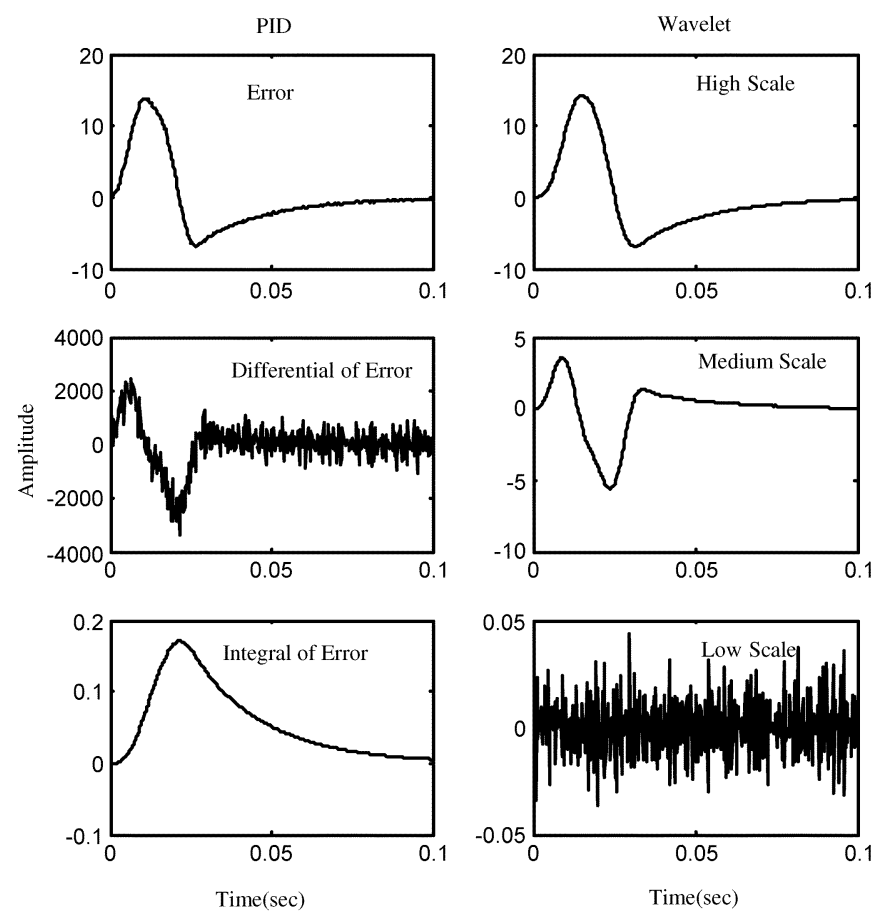

Fig. 4. Comparison of PID and wavelet decomposition of error signal.

these extraneous signals occur in the system. But, in many situations, feedback can reduce the effect of noise and disturbance on the system performance. In practice, disturbance and commands are often low-frequency signals, whereas sensor noises are often high-frequency signals. This makes it difficult to minimize the effect of these uncertainties simultaneously. It is under these conditions that an MRPID performs extremely well. Fig. 4 shows the comparison of signals generated by applying a PID scheme (error, differential of error, and integral of error) to the error signal and a multiresolution decomposition (low scale, medium scale and high scale) of the error signal. The high-scale signal filters out noise and high-frequency distortion from the error signal. Increasing the gain corresponding to high-scale signal $\left(K_{H}\right)$ pushes the control bandwidth and improves the disturbance rejection of the plant. Consider the medium-scale signal in Fig. 4. It approximates the differential of the error signal with low gain and it has a very low noise content compared to differentiation performed using a first-order filter. Such a noise-free differentiation enables us to increase the corresponding gain and add damping to the plant, thereby improving its transient response. The lowest scale signal filters out the noise. By adjusting the lowest scale gain to zero $\left(K_{L}\right)$, we can produce a very smooth control signal and drastically reduce the effect of noise on the plant output. Smooth control effort improves the life of the motor and the overall performance of the plant.

\section{IMPLEMENTATION ISSUES}

There are a number of practical considerations that must be addressed in order to come up with a useful wavelet analysis of the time series applicable to controls. Some of these issues include the type and size of the wavelet to use, how to calculate the instantaneous wavelet transform of a signal when a sample of signal becomes available (for real-time control), the number

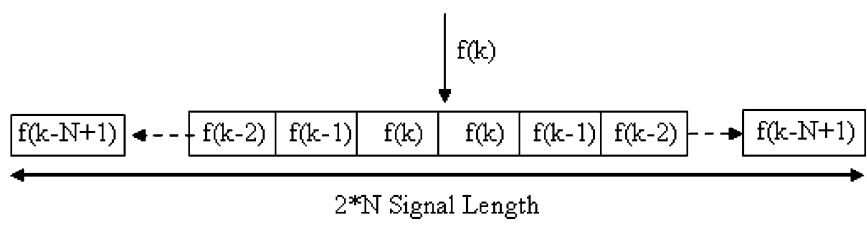

Fig. 5. Signal architecture.

of decomposition levels, and the number of samples to use in the transform.

\section{A. Signal Pipeline Architecture}

Wavelet transform is performed on a bunch of data after they are made available to the processing engine, on account of the noncausal nature of the wavelets. In order to have causal processing, a delay has to be introduced in the channel. This delay is proportional to the number of samples used in the computation. The other issue that further adds to the delay is the ill-conditioning of the data at the boundaries. As control systems require real-time signal processing in order to operate in real time, this delay has been a bottleneck in application of wavelets in controls. Traditionally, researchers have worked with the wavelets on the half axis, which work only on past data or circular data structures. In order to perform multilevel decomposition for real-time operation, a pipeline data architecture was developed. This architecture is illustrated in Fig. 5.

In this scheme, a signal buffer of length $L$ is chosen such that $L=2^{N}$ where $N$ is the number of decomposition levels desired in analysis. Initially, the signal buffer is filled with zeros. When the current ( $k$ th) sample becomes available, it is pipelined into the buffer using the first in first out (FIFO) operation. The signal buffer values are mirrored and appended so as to have the latest data concentrated toward the center. The decomposition algorithm is then performed on the resultant signal buffer. The decomposed $k$ th sample is then available at the center of the signal buffer.

\section{B. Number of Decomposition Levels}

In order to obtain sufficient resolution in both time and frequency, the number of levels $(N)$ that a signal is decomposed depends upon the size of the signal buffer (number of observations in the time series) $(L)$ and the size of the filter $(F)$ used. $N$ is set to be the largest integer satisfying the equation

$$
N \leq=\log _{2}\left(\frac{2^{*} L-1}{F-1}+1\right) .
$$

From the perspective of a control system, this represents the number of tuning, i.e., the gains of the controller.

\section{Selection of Wavelet}

The first problem in constructing a wavelet analysis is the selection of a particular wavelet from amongst all available ones. A reasonable choice depends upon the application at hand. In control application, the objective is to apply wavelet analysis to the error signal. The choice depends on the interplay between a specific analysis goal (such as signal decomposition to separate noise) and the properties we need in a wavelet filter to achieve that goal. 


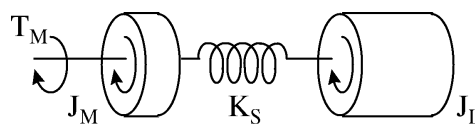

Fig. 6. Simple compliantly coupled motor and load.

It has been found that a wavelet of very short widths can sometimes introduce undesirable artifacts into the resulting analysis, however, they may be desirable on account of their flexibility to provide smaller computational effort and ease in applications involving real-time operations. On the other hand, a wavelet with a large number of coefficients can match the characteristic features in a time series with greater efficiency. Their use can result in more coefficients being unduly influenced by boundary conditions, some decrease in the degree of localization of DWT coefficients, and an increase in computational burden. A reasonable overall strategy is to use the smallest sized filter that gives a reasonable result and be alienable in time (i.e., a phase shift as small as possible). Further details on the selection of wavelets can be found in [10].

\section{Simulation RESUlts of A LOW-FrEQUENCY RESONANCE SYSTEM}

Two different motion control problems were investigated for implementation of the wavelet controller. The first is a velocity control servo system exhibiting low-frequency mechanical resonance, which is discussed in this section, and the second one is a position control system which is discussed in Section VI.

Servo system inertia mismatch, between load and motor, has long been a concern for motion system designer. Most problems of resonance are caused by transmission components and the inertia mismatch between motor and load. For a servo system to operate effectively, servo amplifiers need to be tuned to optimize the response of the system, which includes command response and disturbance rejection. Standard servo control laws are structured for rigidly coupled loads. However, in practical machines, some compliance is always present; this compliance in addition to the inertia mismatch reduces control loop stability margins, forcing servo gains down, which lowers the machine performance. Often, the resulting rigidity of the transmission is so low that instability results when control-law gains are raised to levels necessary to achieve the desired servo performance. The well-known lumped-parameter model [11] for a compliant coupling is shown in Fig. 6 . The motor with inertia $J_{M}$ produces a torque $T_{M}$ which is used to drive a load of inertia $J_{L}$. Equivalent spring constant of the entire system is represented by $K_{S}$.

A schematic diagram of the compliantly coupled mechanism of Fig. 6 is shown in Fig. 7. Here, the equivalent spring constant of the entire transmission is $K_{S}$; also, to represent loss-producing properties, a mechanical damping term is shown producing torque in proportion to velocity differences via crosscoupled viscous damping $b_{s}$.

The transfer function from electromechanical torque $T_{E}$ to motor velocity $V_{M}$ is

$$
\frac{V_{M}}{T_{E}}=\frac{1}{J_{M}+J_{L}} \frac{1}{s} \frac{J_{L} s^{2}+b_{s} s+K_{s}}{\frac{J_{L} J_{M}}{J_{L}+J_{M}} s^{2}+b_{s} s+K_{s}}
$$

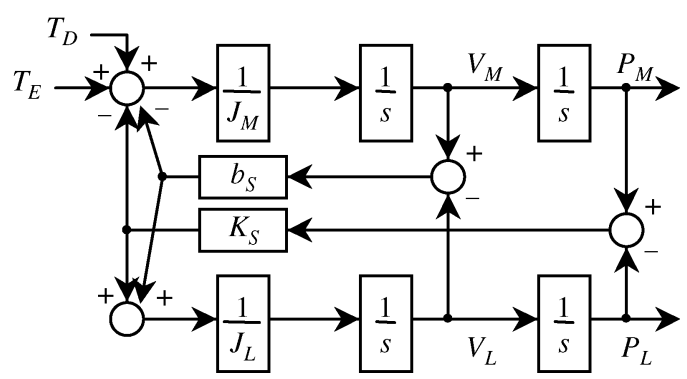

Fig. 7. Block diagram of a compliantly coupled load.

which is a single lumped inertia $1 /\left[\left(J_{M}+J_{L}\right)\right] s$, modified by a bi-linear quadratic or "bi-quad" function. The bi-quad term has its minimum gain at $F_{A R}$ and maximum at $F_{R}$, shown as follows:

$$
\begin{aligned}
F_{A R} & =\frac{1}{2 \pi} \sqrt{\frac{K_{S}}{J_{L}}} \mathrm{~Hz} \\
F_{R} & =\frac{1}{2 \pi} \sqrt{\frac{K_{S}}{\frac{J_{L} J_{M}}{J_{L}+J_{M}}}} \mathrm{~Hz} .
\end{aligned}
$$

The bi-quad term corrupts the plant at and above the antiresonant frequency $F_{\mathrm{AR}}$. The key problem presented by a compliant coupling for low-frequency resonance [11] is the net increase in gain above the resonant frequency $F_{R}$.

The model parameters were selected to produce a resonant frequency which is consistent with machines used in industry. The parameters were $J_{M}=1.8 * 10^{-5} \mathrm{Kg} \cdot \mathrm{m}^{2}, J_{L}=$ $6.3 * 10^{-5} \mathrm{Kg} \cdot \mathrm{m}^{2}, K_{S}=30 \mathrm{Nm} / \mathrm{rad}, K_{T}=0.44 \mathrm{Nm} / \mathrm{rad}$, and $b_{S}=0.005 \mathrm{Nm} \cdot \mathrm{s} / \mathrm{rad}$. This ratio produces a resonance frequency of $233 \mathrm{~Hz}$. The MRPID was used to control the velocity of the resonant plant. Three-level decomposition of the error signal was done resulting in a high-scale, low-scale, and two intermediate-scale signals. Each of these signals were scaled by their respective gains $\left(K_{H}, K_{L}, K_{M 1}\right.$, and $K_{M 2}$ ) and added to compute the control signal using (13). Comparison of simulation results for a PI controller using a noninteracting PID equation and an MRPID are shown in Fig. 8. The PI controller was tuned with $K_{P}=2.5$ and $K_{I}=15 \mathrm{~ms}$. MRPID was tuned with $K_{H}=0.025, K_{M 1}=0.2, K_{M 2}=0.2$ and $K_{L}=0$. It can be seen that the MRPID performs slightly better than a PI in transient response, however, it provides much better noise rejection, as can be seen from Fig. 9. This is largely obtained by simply setting the low-scale gain $\left(K_{L}\right)=0$. Noise is an important factor that effects control system performance. It is a well-established fact that noise restricts the bandwidth of the control system and reduces system stability. Therefore, using an MRPID controller offers the distinct advantage of improving system bandwidth and stability.

\section{HARDWARE RESUltS OF POSITION CONTROL SySTEM}

In a typical application using a motor as the power source, the transfer function from input current $(u)$ to output position $(y)$ can be modeled as

$$
G(s)=\frac{b}{s(J s+c)}
$$




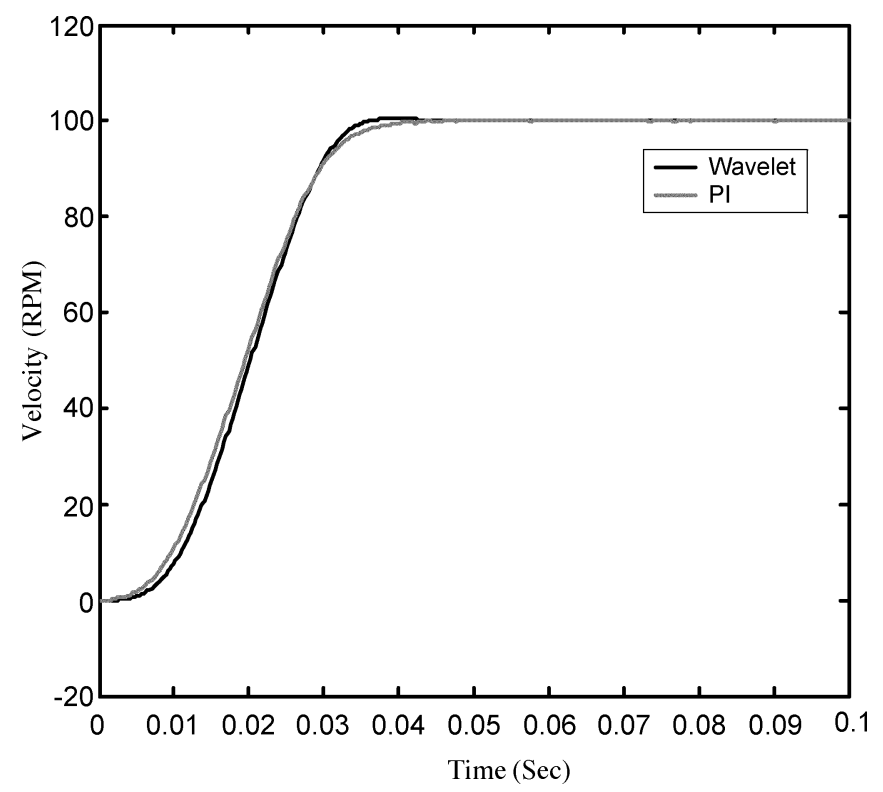

Fig. 8. Simulation results using an MRPID.

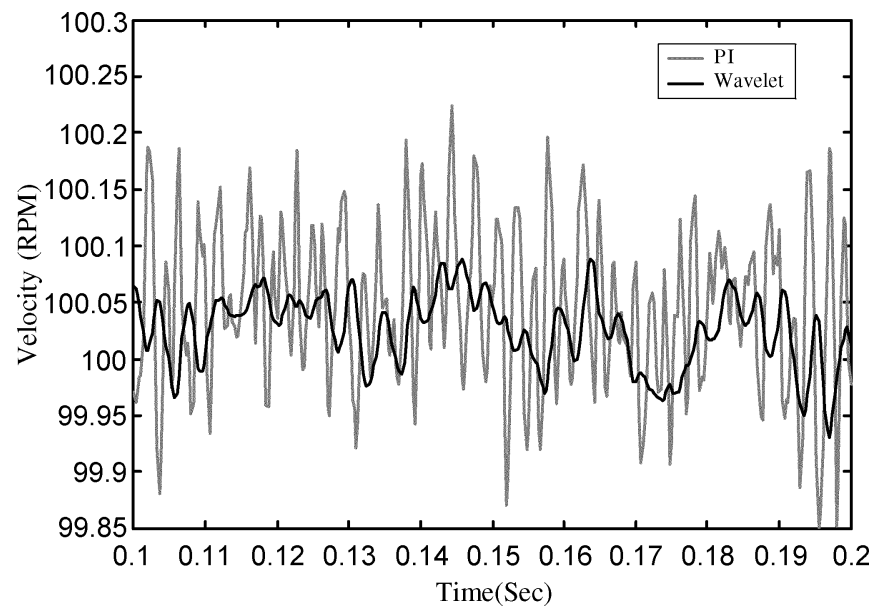

Fig. 9. Effect of noise during steady-state response.

where $b$ is the torque constant, $J$ is the total inertia of motor and load, and $c$ is the viscous friction coefficient. The control objectives are rotating the load one revolution in one second with no overshoot. The experimental setup includes a PC-based control platform and a dc brushless servo system made by ECP (Model 220). The servo system includes two motors (one as an actuator and the other as the disturbance source), a power amplifier, and an encoder, which provides the position measurement. The inertia, friction, and backlash are all adjustable.

A Pentium 133-MHz PC running in DOS is programmed as the controller. It contains a data-acquisition board to read the position encoder output in the servo system. The sampling frequency is $400 \mathrm{~Hz}$. As shown in Fig. 10, the PC performs the position control of the load disc. The position signal is read into the microcomputer via the counter board and the control signal is output to the motor drive via a digital-to-analog controller (DAC). With $T_{D}$ as the torque disturbance, the mathematical model was derived and verified in a hardware test as

$$
\ddot{y}=\left(-1.41 \dot{y}+23.2 T_{D}\right)+23.2 u .
$$

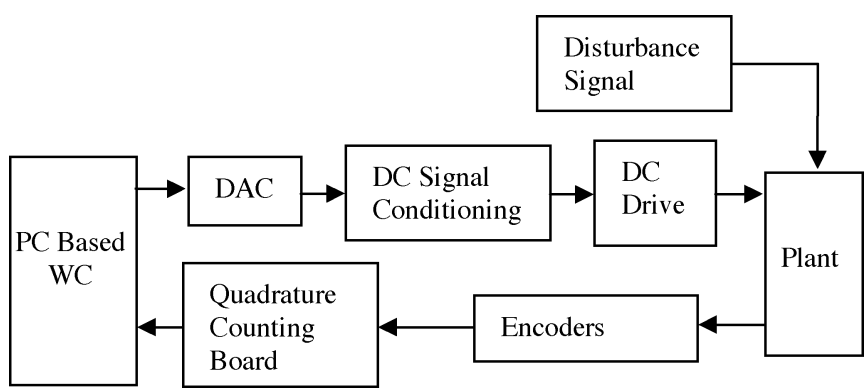

Fig. 10. Block diagram of a dc brushless servo system.

The PID and MRPID control algorithms were written in C language. The subband coding scheme which uses the filters $h(k)$ and $g(k)$ was used to compute the wavelet decomposition. Both of the filters had eight coefficients and the error signal buffer of size 32 was used in the implementation. Three-level decomposition of error signal was done, and the resulting four gain parameters were tuned to generate the control signal $u$ using (13). At nominal plant operation, the PID controller was tuned with $K_{P}=2.8, K_{I}=2.4$, and $K_{D}=1.8$, using a parallel-type PID equation, while the MRPID was tuned with $K_{H}=7.5, K_{M 1}=50, K_{M 2}=40$, and $K_{L}=0$. The plant was then tested with varying test conditions to check the robustness of the controller.

\section{A. Disturbance Rejection}

In order to investigate the disturbance rejection feature of the MRPID, a step torque disturbance was applied using the second motor in the servo system. This occurs after the system reaches the steady state, as shown in Fig. 11. Compared to the PID, the position output recovers from the disturbance much faster in the MRPID on account of its ability to accommodate higher gains. The disturbance settling time is almost five times less than that of a PID. Furthermore, it can be seen that the control signal of a MRPID is smoother and the steady-state error much smaller than that obtained by using a PID.

\section{B. Experimental Results}

To verify the effectiveness of the MRPID, a series of experiments were carried out. These include the following.

1) changing the set-point from 1 to 10 revolutions;

2) increasing the inertia by $125 \%$ (adding two $0.5-\mathrm{kg}$ weights to the disc at a radius of $7.5 \mathrm{~cm}$ );

3) increasing the friction by adjusting the rubbing screw in the test setup;

4) introducing $30 \%$ torque disturbance using the disturbance motor.

For comparison, both the PID and MRPID are tested for each scenario, and the results are evaluated in terms of overshoot, settling time, steady-state error, and the rms error. With $P$ number of samples and $v(t)$ its mean, the rms error is given by

$$
L(e)=\sqrt{\frac{\sum_{P}(v(t)-y(t))^{2}}{P} .}
$$

The results from these tests are listed in Table II. In general, the MRPID performs better than the PID. In particular, when 

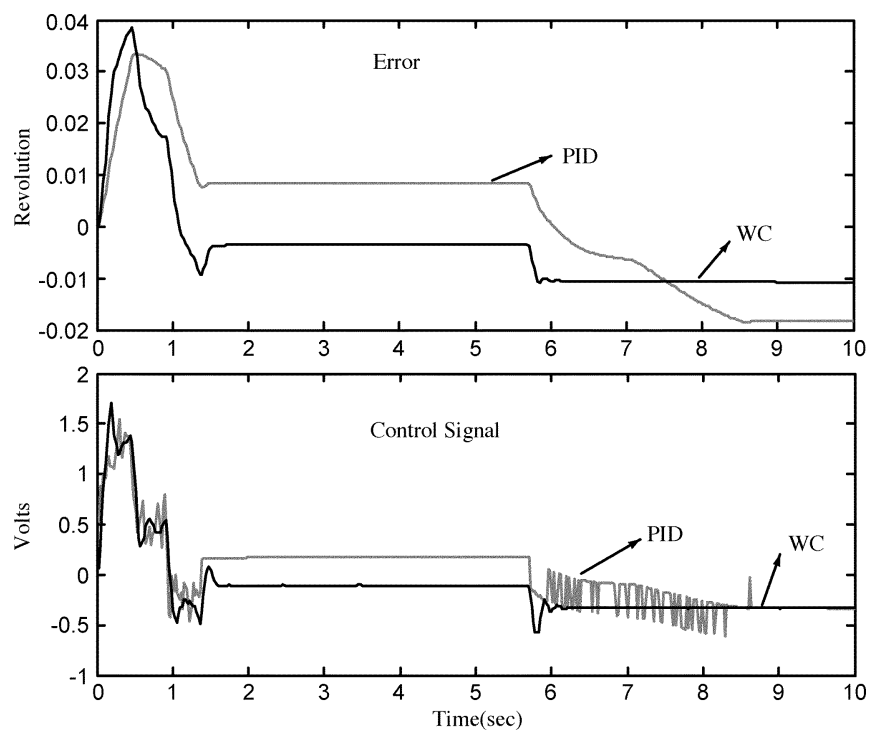

Fig. 11. MRPID and PID responses to a torque disturbance.

TABLE II

EXPERIMENTAL RESULTS USING PID AND WAVELET SCHEMES

\begin{tabular}{lcccc}
\hline \hline & $\begin{array}{l}\text { Over- } \\
\text { Shoot(\%) }\end{array}$ & $\begin{array}{l}\text { Settling } \\
\text { Time(sec) }\end{array}$ & $\begin{array}{l}\text { SS Error } \\
\text { (Rev.) }\end{array}$ & $\begin{array}{l}\text { L(e) } \\
\text { (rad) }\end{array}$ \\
\hline \hline Nominal Case & 0.67 & 1.25 & 0.0083 & 0.0679 \\
\hline 10 Rev. & 0.74 & 12.24 & 0.0771 & 0.2131 \\
\hline Friction Exerted & 0.00 & 1.26 & 0.0372 & 0.0810 \\
\hline Load Added & 0.00 & 1.27 & 0.0107 & 0.0978 \\
\hline $30 \%$ Torque Dist. & N/A & 2.56 & 0.0183 & 0.1372 \\
\hline \hline & & MRPID & & \\
\hline Nominal Case & 0.85 & 1.21 & 0.0034 & 0.0560 \\
\hline 10 Rev. & $\mathbf{0 . 0 0}$ & 12.19 & $\mathbf{0 . 0 1 1 2}$ & 0.1446 \\
\hline Friction Exerted & 0.04 & 1.22 & $\mathbf{0 . 0 0 1 4}$ & 0.0620 \\
\hline Load Added & 0.21 & 1.20 & 0.0018 & 0.0614 \\
\hline $30 \%$ Torque Dist. & N/A & $\mathbf{0 . 5 9}$ & 0.0108 & 0.1118 \\
\hline \hline
\end{tabular}

the number of revolutions is changed from a nominal value of 1 to 10 , the percentage overshoot using the PID persists; however, in the case of the MRPID, it goes to zero. Furthermore, the steady-state error in the case of the PID increased by almost 10 times due to this change, but it only increased by three times in the case of the MRPID. By assigning low gain to high-frequency component $\left(K_{L}\right)$, we can actively remove noise from the control signal. Furthermore, in the absence of noise, gain corresponding to low-frequency component $\left(K_{H}\right)$ can be tuned to a higher value. This improves the transient response and disturbance rejection of the plant, as can be seen from Fig. 11 .

\section{CONCLUSION}

A generalized multiresolution PID controller based on the wavelet transforms is proposed and tested. Just like a conventional PID controller, the MRPID is intuitive and effective. The controller gains have an explicit relationship with the characteristics of the error signal, which makes tuning of the controller insightful. The wavelet decomposition, which represents the error signal at different scales, provides higher resolution representation of the error signal than the one used in the existing PID and is the basis of the new controller design. Implementation of the wavelet-based multiresolution controller in hardware shows promising results, particularly in its ability to provide smooth control effort and better noise and disturbance rejection compared to the existing PID controller. More research efforts are underway to fully explore the potential of this new design concept.

\section{REFERENCES}

[1] G. Stephanopoulos, O. Karsligil, and M. Dyer, "Multi-scale aspects in model-predictive control," J. Process Contr., vol. 10, pp. 275-282, 2000.

[2] I. Daubechies, Ten Lectures on Wavelets, ser. CBMS-NSF Regional Conf. Series Appl. Math.: SIAM, 1992.

[3] S. G. Mallat, "A theory of multi-resolution signal decomposition: The wavelet representation," IEEE Trans. Pattern Anal. Mach. Intell., vol. 11, no. 7, pp. 674-693, Jul. 1989.

[4] S. Ungarala and B. R. Bakshi, "Multiscale bayesian estimation and data rectification," in Wavelets in Signal and Image Processing, A. Petrosian and F. Meyer, Eds. New York: Kluwer, 2001.

[5] S. Parvez and Z. Gao, "A novel controller based on multi-resolution decomposition using wavelet transforms," in Proc. ISA Tech. Conf. Annual Meeting, Chicago, IL, 2002, CD-ROM.

[6] I. Daubechies, "The wavelet transform, time-frequency localization and signal analysis," IEEE Trans. Inf. Theory, vol. 36, no. 5, pp. 961-1005, Sep. 1990

[7] C. K. Chui, Wavelets: A Tutorial in Theory and Applications. Boston, MA: Academic, 1992

[8] N. Minorsky, "Directional stability and automatically steered bodies," $J$. Amer. Soc. Nav. Eng., vol. 34, p. 280, 1922.

[9] Z. Gao, "From linear to nonlinear control means: a practical progression," ISA Trans., vol. 41, no. 2, pp. 177-189, Apr. 2002.

[10] S. Parvez, "Advanced control techniques for motion control problem," $\mathrm{Ph} . \mathrm{D}$. dissertation, Cleveland State Univ., Cleveland, OH, Dec. 2003.

[11] G. Ellis and R. D. Lorenz, "Resonant load control methods for industrial servo drives," in Conf. Rec. IEEE-IAS Annu. Meeting, vol. 3, Oct. 8-12, 2000, pp. 1438-1445. 